\title{
Separation of stereoisomeric mixtures of nafronyl as a representative of compounds possessing two stereogenic centers by coupling crystallization diastereoisomeric conversion and chromatography
}

\author{
Dawid Kiwala ${ }^{\mathrm{a}}$, Maksymilian Olbrycht ${ }^{\mathrm{b}}$, Maciej Balawejder, ${ }^{\mathrm{c},}$, Wojciech Piątkowski ${ }^{\mathrm{b}}$, \\ Andreas Seidel-Morgenstern ${ }^{\mathrm{a}, \mathrm{d}}$, Dorota Antos, ${ }^{\mathrm{b}, *}$ \\ ${ }^{a}$ Max Planck Institute for Dynamics of Complex Technical Systems, 39106 Magdeburg/DE \\ ${ }^{b}$ Department of Chemical and Process Engineering, Faculty of Chemistry, Rzeszow University of Technology, \\ 35-959 Rzeszow/PL \\ ${ }^{c}$ Chair of Chemistry and Food Toxicology, University of Rzeszow, 35-959 Rzeszow/PL \\ ${ }^{d}$ Institute of Process Engineering, Faculty of Process and Systems Engineering, Otto von Guericke University \\ Magdeburg, 39106 Magdeburg/DE
}

ABSTRACT: A procedure for the isolation of the most biologically active component of a stereoisomeric mixture of nafronyl - 2-(diethylamino)ethyl 3-(naphthalen-1-yl)-2-

((tetrahydrofuran-2-yl)methyl)propanoate, has been proposed. The molecule of nafronyl is a representative of compounds possessing two stereogenic centers, which may be produced in the form of a quaternary mixture that contains two pairs of racemates being diastereoisomers of one another. The components of such stereoisomeric mixtures usually differ in pharmacological activity, therefore, there is an interest in developing efficient methods for their resolution. The method suggested in this study comprised two sequential separation processes, including multistage cross-current crystallization and chiral chromatography. Crystallization was employed to enrich the raw material mixture with the target racemate, which contained the stereoisomer exhibiting the highest biological activity. To intensify the process, the mother liquors depleted with the target racemate were subjected to fast basecatalyzed diastereoisomeric conversion in melt, which provided equimolar mixtures of all four stereoisomers. The coupling of cross-current crystallization and diastereoisomeric conversion could improve yield of crystallization from $29 \%$ up to $84 \%$. The purified racemate was further processed by chromatography to isolate finally the most active stereoisomer with $99 \%$ purity and total yield of $84 \%$, at a relatively high throughput.

\footnotetext{
* Corresponding authors:

dorota.antos@prz.edu.pl, tel.: +48 178651853; fax: +48 178543655

maciejb@univ.rzeszow.pl, tel.: +48 178621745; fax: + 48178721265
} 
For Table of Contents Use Only

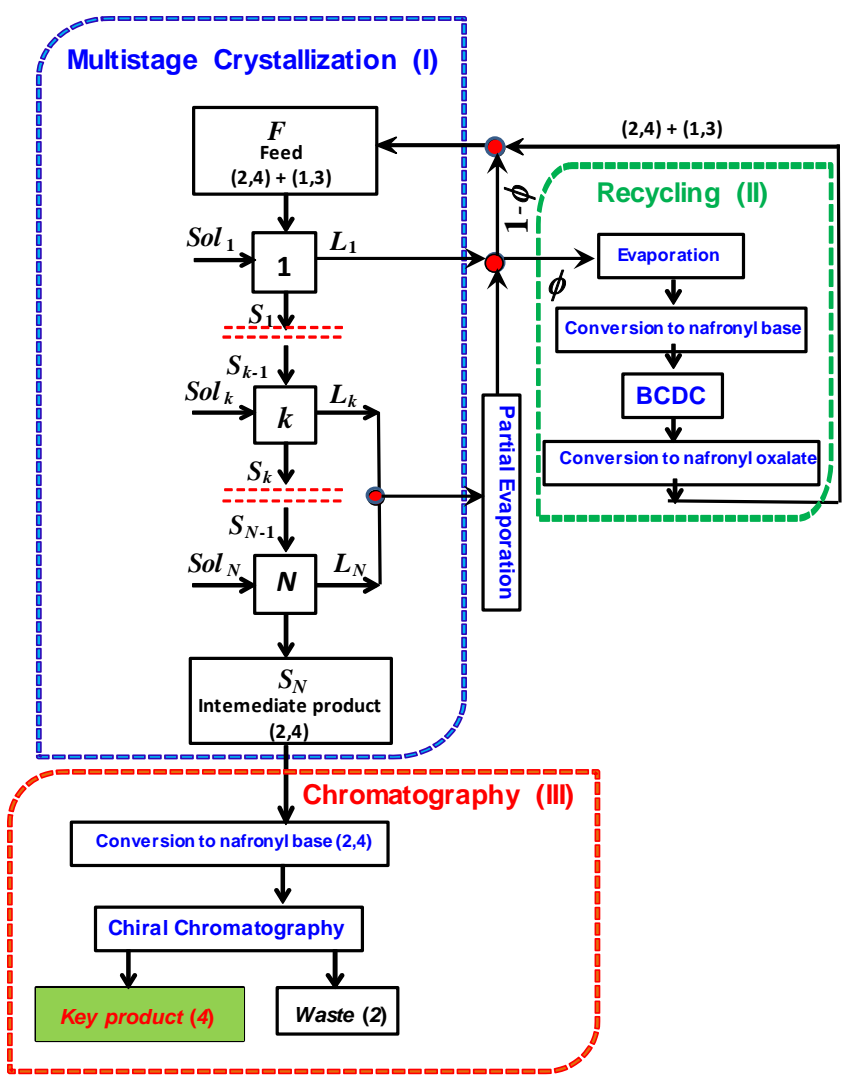


Keywords: chiral separation, nafronyl, multistage crystallization, chiral chromatography, diastereoisomeric conversion, solid solution. 


\section{INTRODUCTION}

The worldwide interest in optically pure compounds has stimulated increase in production of single enantiomers, which displaced previously marketed racemic mixtures in many areas of medicine and pharmacy. ${ }^{1-4}$ This trend emerged as a result of increasing awareness of individual biological properties for opposite enantiomers in racemic drugs. ${ }^{5-8}$ Two approaches can be used to produce single enantiomers, which involve either resolution of racemates or stereoselective synthesis from achiral material. ${ }^{9}$ Nevertheless, manufacturing racemates is usually more economical than stereoselective synthesis of enantiomers. ${ }^{10}$ It particularly holds true when efficient methods for separating racemic mixture are available, which provide high yield and optical purity, as well as the possibility of recycling of unwanted enantiomer by implementing a racemization procedure. The latter allows overcoming the problem associated with the yield limitation in chiral resolutions, where unwanted enantiomers are wasted. ${ }^{8-11}$

A number of separation techniques can be used for the optical resolution. Among them chiral chromatography and enantioselective crystallization are considered the most straightforward, economical and easiest to perform on a large scale. ${ }^{9,12-14}$

In the so-called classical resolution method, the enantiomers are converted into a pair of diastereomeric salt that possess the same chemical formula but have different physical properties. ${ }^{15}$ The diastereoisomers are then separated using crystallization, taking advantage of the difference in solubility between the two components. ${ }^{16}$ Typically, crystallizationinduced diastereoisomeric transformation is realized by formation of ionic or coordinate bonds with a single-enantiomer resolving agent, i.e., a diasteromeric salt pair, by transformation of covalent bonds (epimerization), or by creating new stereogenic center.

In most cases, racemate containing the equilibrating center (i.e., the center with undefined configuration) is combined with a molecule with the directing center (i.e., the center with defined stereochemical configuration), which influences on the manner of ordering the molecules in the crystalline phase, hence, on the resolution of diastereoisomers. ${ }^{17}$

However, literature data on the production and separation of compounds having two stereogenic centers is scarce. The increased number of stereogenic centers in the molecule complicates the synthesis of single enantiomer, therefore, a non-selective synthesis is often applied, which generates stereoisomeric mixtures. Such mixtures consist of two racemates, in which the equilibrating center is covalently linked to the directing center. The isolation of a target stereoisomer out of such a mixture is a challenging task. Thus, research on a generic 
procedure for the realization of resolution process is of major importance and may contribute in progress in pharmacology and organic process development.

In this study preliminary attempts have been made to tackle that separation problem. The subject of the study was a compound called nafronyl or naftidrofuryl that is 2-(diethylamino)ethyl 3-(naphthalen-1-yl)-2-((tetrahydrofuran-2-yl)methyl)propanoate, with the chemical formula $\mathrm{C}_{24} \mathrm{H}_{33} \mathrm{NO}_{3}$. The chemical structure is depicted in Figure 1.
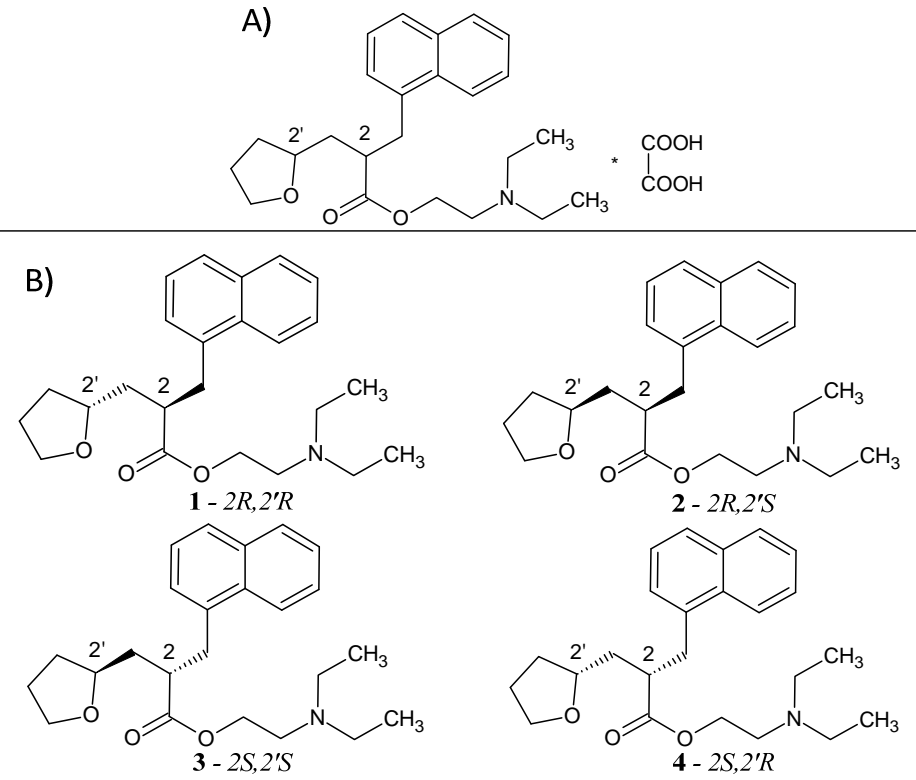

Figure 1. Chemical structure of nafronyl. A) Nafronyl oxalate; B) nafronyl free base stereoisomers.

Nafronyl is marketed in the form of addition salt with oxalic acid (Figure 1A) under trade names such as, e.g.,Praxilene and Dusodril. The drug is commercially available as a mixture of four stereoisomers. It is used in medicine in the form of oxalate salt for the treatment of vascular diseases, including intermittent claudication, stroke and dementia. The pharmacological properties of nafronyl have been described in detail by Goldsmith and Wellington ${ }^{18}$ and Barradell and Broqden. ${ }^{19}$

Due to the existence of two stereogenic centers in the position 2 of the propionic acid backbone and in the position $2^{\prime}$ of the tetrahydrofuryl carbon atom, the molecule has four stereoisomers, i.e., it forms two racemates which comprise enantiomeric pairs: ${ }^{20}$ $\left(2 R, 2^{\prime} R\right):\left(2 S, 2^{\prime} S\right)$ and $\left(2 R, 2^{\prime} S\right):\left(2 S, 2^{\prime} R\right)$. A recent study revealed that each of isomers exhibited individual pharmacodynamic properties, and the isomer having $\left(2 S, 2^{\prime} R\right)$ configuration contributed most to the therapeutic efficacy. ${ }^{21}$

Several methods have been suggested to produce single stereoisomers of nafronyl, ${ }^{21-23}$ where a desired configuration was obtained in a combination of stereoselective synthesis that provided the target pair of diastereoisomers, with diasteroisomeric crystallization of the post- 
reaction mixture. ${ }^{21-23}$ Nevertheless, the reported techniques required a long chemical procedure with a low efficiency and high cost associated with using chiral reagents in stereoselective synthesis. Therefore, a concept of resolving stereoisomeric mixtures, which are obtained via relatively simple non-selective synthesis, by a combination of crystallization and chromatography, might be a promising alternative.

In this study we used diastereoisomeric crystallization to isolate the racemate $\left(2 R, 2^{\prime} S\right):\left(2 S, 2^{\prime} R\right)$, which comprised the most biologically active stereoisomer $\left(2 S, 2^{\prime} R\right)$ from a quaternary stereoisomeric mixture. Prior to the process realization, solid-liquid phase equilibrium (SLE) was quantified for stereoisomeric mixtures of nafronyl oxalate salt in acetone. The SLE pattern indicated that stereoisomers of nafronyl oxalate form solid solution in the crystalline phase, therefore, resolution of the stereoisomeric mixture required multistage crystallization. The conversion of nafronyl oxalate to another salt (e.g., nafronyl nitrate) has not improved stereoselectivity of the solution. ${ }^{23}$ On the basis of the SLE data batchwise multistage cross-current crystallization process was designed. To increase yield of the process the mother liquor, which was depleted with the target racemate, was transferred into an equimolar mixture of all stereoisomers by a fast base-catalyzed reaction in melt, ${ }^{24}$ and recycled to the crystallization process. In this study the reaction was based on diastereoisomeric conversion of $\alpha$-proton on 2 position in nafronyl molecule. The basecatalyzed diastereoisomeric conversion (BCDC) was performed at high temperature on nafronyl free base obtained after a conversion of nafronyl oxalate salt in the presence of sodium hydride as a catalyst. Because nafronyl free base occurs in a liquid form, it had to be converted back to the oxalate salt; the obtained crystalline product of nearly equimolar content of stereoisomers could be reused in the crystallization process. The enantiomeric mixture $\left(2 R, 2^{\prime} S\right):\left(2 S, 2^{\prime} R\right)$ with HPLC area purity about $99 \%$, which was obtained from the crystallization process, was further processed using chromatography on a chiral stationary phase (CSP), to isolate the target stereoisomer $\left(2 S, 2^{\prime} R\right)$ with a high purity, yield, at a high column load.

\section{CHARACTERIZATION OF NAFRONYL OXALATE SALT}

\subsection{HPLC analysis}

The qualitative and quantitative analysis of nafronyl and its derivatives were performed using a HPLC system (section 5.2.1). To measure optical rotation for each 
stereoisomer and identify the elution order, the HPLC system was additionally equipped with a polarimeter.

The polarimeter signal recorded during chromatographic elution was compared to the optical rotation data for nafronyl stereoisomers reported by Descours, et al. ${ }^{22}$ It has been confirmed that the stereoisomers $\left(2 R, 2^{\prime} R\right)$ and $\left(2 S, 2^{\prime} S\right)$ form an enantiomeric pair with the ratio 1:1 (i.e., racemate), as they exhibit the same value of the optical rotation, but with the opposite sign. Consequently, the stereoisomers $\left(2 R, 2^{\prime} S\right)$ and $\left(2 S, 2^{\prime} R\right)$ form the second racemate containing the target $\left(2 S, 2^{\prime} R\right)$. The elution order of the isomers under the measurement conditions was: $\left(2 R, 2^{\prime} R\right)<\left(2 R, 2^{\prime} S\right)<\left(2 S, 2^{\prime} S\right)<\left(2 S, 2^{\prime} R\right)$. A typical chromatogram of a stereoisomeric mixture of nafronyl oxalate salt is presented in Figure 2. For the sake of simplicity, the following numbers were assigned to each of stereoisomers: $\left(2 R, 2^{\prime} R\right) \equiv \mathbf{1},\left(2 R, 2^{\prime} S\right) \equiv \mathbf{2},\left(2 S, 2^{\prime} S\right) \equiv \mathbf{3},\left(2 S, 2^{\prime} R\right) \equiv \mathbf{4}$ (target).

These numbers are further used to identify the stereoisomers. Two peaks that appear at the beginning of the chromatogram are attributed to the signal difference between the eluent and the solvent used for the sample preparation. No additional impurity peaks were observed.

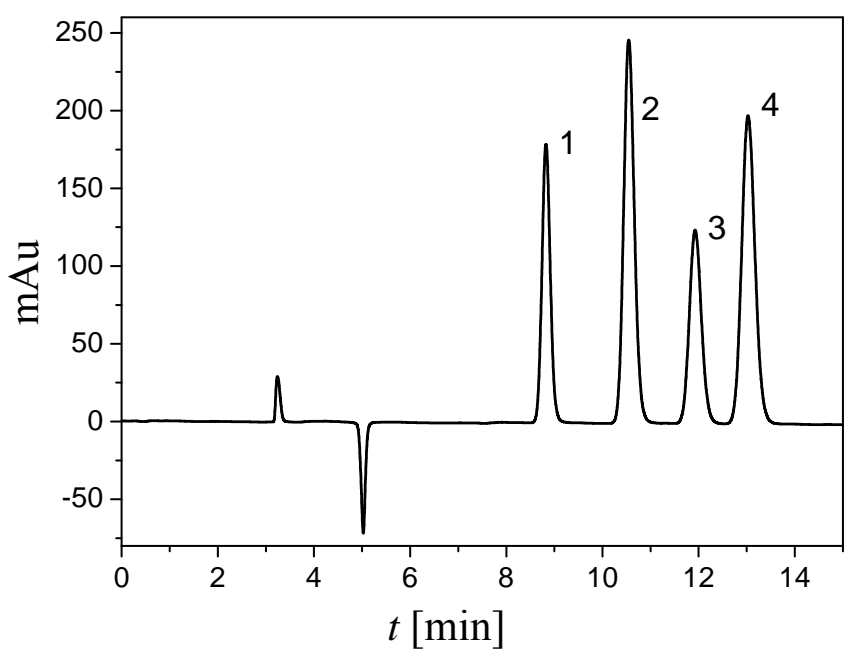

Figure 2. Chromatogram of a stereoisomeric mixture of nafronyl oxalate salt containing two pairs of racemates each with two enantiomers $\mathbf{1 , 3}$ and $\mathbf{2 , 4}$ (for the measurement conditions see section 5.2.1).

\subsection{SLE measurements}

The SLE measurements were performed in acetone under isothermal conditions according to the procedure reported in section 5.2.4. The equilibrium content of the target racemate 2,4 in the solid phase, $x_{2,4}^{S}$, ranged from $0.63[\mathrm{~g} / \mathrm{g}]$, which corresponded to the raw material (feed), to about $1[\mathrm{~g} / \mathrm{g}]$, corresponding to the pure racemate $\mathbf{2 , 4}$. 
The results obtained are summarized in Table 1 and Figures $3 \mathrm{~A}-3 \mathrm{C}$, where the following process performance indicators are presented:

- diastereoisomeric excess of the desired racemate, $d e_{2,4}$, defined as follows:

$$
d e_{2,4}=\frac{\left(x_{2,4}^{S}-x_{1,3}^{S}\right)}{\left(x_{2,4}^{S}+x_{1,3}^{S}\right)}
$$

where $x_{2,4}^{S}, x_{1,3}^{S}$ denote mass fractions of the racemate $\mathbf{2 , 4}$ and $\mathbf{1 , 3}$, respectively;

- $\quad$ separation yield for $k$-th stage:

$$
Y=\frac{S_{k} x_{2,4, k}^{S}}{S_{k-1} x_{2,4, k-1}^{S}}
$$

- total yield for the whole operation:

$$
Y_{\text {total }}=\frac{S_{N} x_{2,4, N}^{S}}{F x_{2,4, F}^{S}}
$$

where $Y_{k}$ is separation yield of each $k$-th stage; $S_{k-1}, S_{k}$ is the mass of the crystalline phase withdrawn from the stage $k-1$, and delivered into the corresponding subsequent stage $k$, respectively; $x_{2,4, k-1}^{S}, x_{2,4, k}^{S}$ is the mass fraction of the racemate $\mathbf{2 , 4}$ in the corresponding solid phases; for the first stage $(k=1)$ or for single-stage crystallization the values of $S_{k-1}$ and $x_{2,4, k-1}^{S}$ are equivalent to mass of feed, $F$, and the mass fraction of the racemate 2,4 in feed, $x_{2,4, F}^{S}$, respectively; $Y_{\text {total }}$ is yield of the whole crystallization process including $N$ stages.

- distribution coefficient of the target racemate:

$$
k_{2,4}=x_{2,4}^{S} / \bar{x}_{2,4}^{L}
$$

where $\bar{x}_{2,4}^{L}=x_{2,4}^{L} /\left(x_{2,4}^{L}+x_{1,3}^{L}\right)$ is the solvent-free fraction in the liquid phase.

- solvent consumption defined as the mass ratio of solvent, Sol, and crystalline phase $S_{k-1}$, delivered into each $k$-th step:

$$
S C=\frac{S o l}{S_{k-1}}
$$

Figure $3 \mathrm{~A}$ presents the ternary phase diagram, where the equilibrium compositions of the solid and liquid phases were linked by tie lines. Because of identical properties of enantiomers in achiral environment the liquid mixture of two racemates and a solvent can be considered as a pseudo ternary mixture. The course of tie lines is characteristic for solid solution systems with minimum of solubility for pure target racemate $\mathbf{2 , 4}$ in the solid phase. ${ }^{25}$ 
Additionally, the SLE data were depicted on the distribution phase diagram, where the mass fraction of the racemate $\mathbf{2 , 4}$ in the solid phase was plotted against its mass fraction in the solvent-free mother liquor $\left(\bar{x}_{2,4}^{L}\right.$, Figure 3B). It can be observed that within the investigated SLE range the enrichment of the target racemate occurred in the crystalline phase. The highest process efficiency, which corresponds to the maximum of yield, $Y$ (Eq.2), is graphically illustrated by the point located at the largest distance from the diagonal. That maximum is achieved for $\bar{x}_{2,4}^{L}$ equal to about 0.37 [g/g]. The slope of the distribution curve represents the phase distribution coefficient, $k_{2,4}$ (Eq.4). It decreases with increase in concentration of the target racemate and tends to zero as $\bar{X}_{2,4}^{L}$ goes to 1 . The changes of phase distribution coefficient, yield and solvent consumption (SC, Eq.5) versus the liquid phase composition are depicted in Figure 3C.

Table 1. SLE data for stereoisomeric mixtures of nafronyl oxalate salt with different $d e_{2,4}$ (Eq.1). $S, L$ are the mass of crystalline and liquid phase at equilibrium conditions, respectively.

\begin{tabular}{|c|c|c|c|c|c|c|c|c|c|c|}
\hline \multicolumn{4}{|c|}{ Inlets } & \multicolumn{9}{c|}{ Outlets } \\
\hline \multicolumn{4}{|c|}{ FEED } & \multicolumn{3}{c|}{ SOLID PHASE } & \multicolumn{3}{|c|}{ MOTHER LIQUID } \\
\hline $\begin{array}{c}F \\
{[\mathrm{~g}]}\end{array}$ & $\begin{array}{c}S C \\
{[\mathrm{~g} / \mathrm{g}]}\end{array}$ & $\begin{array}{c}x_{2,4} \\
{[\mathrm{~g} / \mathrm{g}]}\end{array}$ & $\begin{array}{c}d e_{2,4} \\
{[\%]}\end{array}$ & $\begin{array}{c}S \\
{[\mathrm{~g}]}\end{array}$ & $\begin{array}{c}x_{2,4}^{S} \\
{[\mathrm{~g} / \mathrm{g}]}\end{array}$ & $\begin{array}{c}d e_{2,4} \\
{[\%]}\end{array}$ & $\begin{array}{c}L \\
{[\mathrm{~g}]}\end{array}$ & $\begin{array}{c}x_{1,3}^{L} \\
{[\mathrm{~g} / \mathrm{g}]}\end{array}$ & $\begin{array}{c}x_{2,4}^{L} \\
{[\mathrm{~g} / \mathrm{g}]}\end{array}$ & $\begin{array}{c}d e_{2,4} \\
{[\%]}\end{array}$ \\
\hline 0.5 & 7.91 & 0.6300 & 26 & 0.3916 & 0.7134 & 43 & 4.0634 & 0.0177 & 0.0070 & -43 \\
\hline 0.4 & 9.89 & 0.7000 & 40 & 0.3138 & 0.7788 & 56 & 4.0412 & 0.0147 & 0.0067 & -38 \\
\hline 0.4 & 9.89 & 0.7500 & 50 & 0.3377 & 0.8271 & 65 & 4.0173 & 0.0097 & 0.0059 & -24 \\
\hline 0.4 & 11.87 & 0.8000 & 60 & 0.3263 & 0.8669 & 73 & 4.8197 & 0.0087 & 0.0067 & -13 \\
\hline 0.2 & 15.82 & 0.8250 & 65 & 0.1626 & 0.8927 & 79 & 3.2014 & 0.0065 & 0.0053 & -10 \\
\hline 0.2 & 47.46 & 0.8500 & 70 & 0.1197 & 0.9392 & 88 & 9.5723 & 0.0027 & 0.0057 & 36 \\
\hline 0.2 & 63.28 & 0.9000 & 80 & 0.1186 & 0.9615 & 92 & 12.7374 & 0.0013 & 0.0051 & 59 \\
\hline 0.2 & 63.28 & 0.9250 & 85 & 0.1252 & 0.9711 & 94 & 12.7308 & 0.0009 & 0.0050 & 70 \\
\hline 0.2 & 63.28 & 0.9500 & 90 & 0.1159 & 0.9796 & 96 & 12.7401 & 0.0007 & 0.0059 & 79 \\
\hline 0.2 & 71.19 & 0.9750 & 95 & 0.1180 & 0.9904 & 98 & 14.3200 & 0.0004 & 0.0053 & 86 \\
\hline 0.2 & 71.19 & 0.9950 & 99 & 0.1194 & 1.0000 & 100 & 14.3186 & 0.0001 & 0.0055 & 96 \\
\hline
\end{tabular}




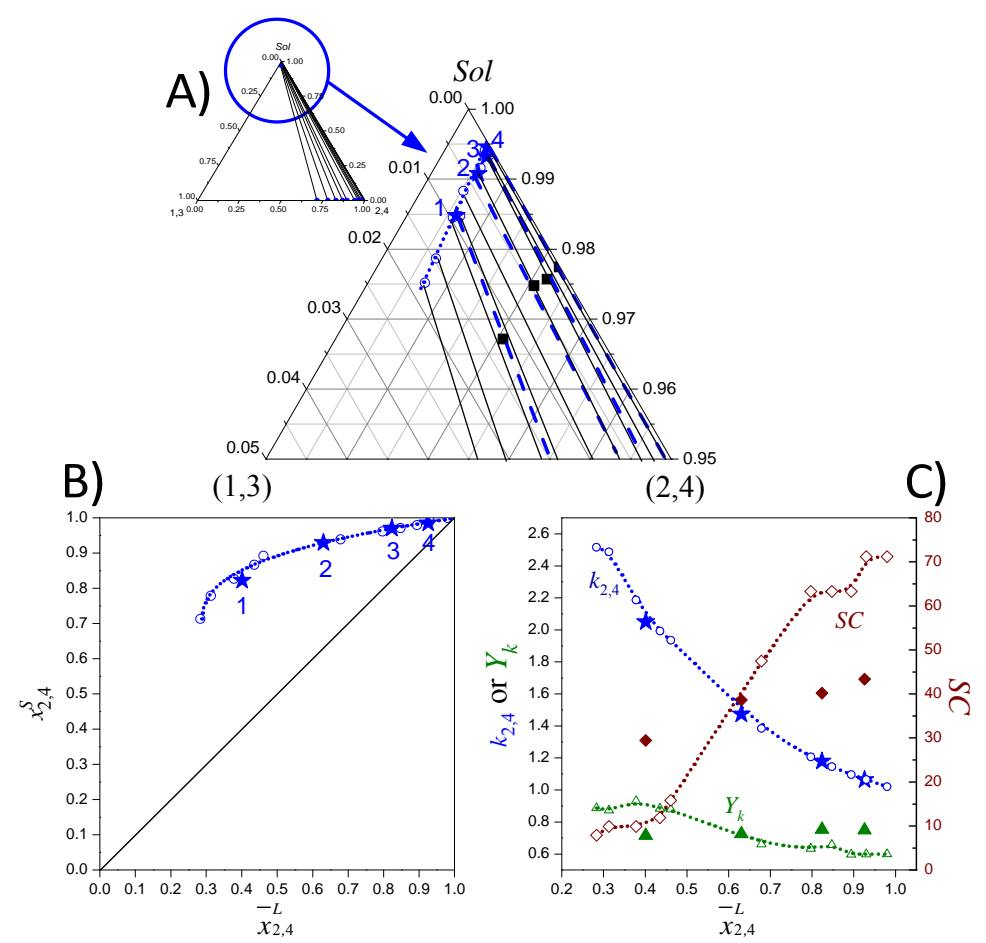

Figure 3. SLE data of the ternary mixture of the racemate 1,3 and 2,4 of nafronyl oxalate salt in acetone at $20^{\circ} \mathrm{C}$. A) Ternary phase diagram of stereoisomeric mixture with an enlargement of the upper part of the triangle, solid lines - tie lines for the SLE data, dashed lines - tie lines for multistage crystallization experiment (with four stages $1,2,3,4$ ), open circles - SLE data, solid circles - multistage crystallization experiment, solid squares - mixing points, dotted line - to guide eye for the solubility pattern; B) distribution of the racemate 2,4 between solid and liquid phase, symbols as in A; C) dependence of the distribution coefficient $k_{2,4}$, yield $Y$, and the solvent consumption, $S C$, for each $k$-th experiment, versus $\bar{x}_{2,4}^{L}$, symbols as in A.

\subsection{DSC measurements}

To analyze the possibility of solvation or a phase transition in the crystalline lattice of stereoisomeric mixtures of nafronyl oxalate salt, differential scanning calorimetry (DSC) was used (section 5.2.6). Thermograms were recorded for several samples with different diastereoisomeric enrichment, $d e_{2,4}$, which were acquired from the crystalline phases obtained in the SLE experiments. Additionally, samples of the solid residues of evaporated mother liquors were also acquired and examined.

According to the DSC data, the melting point of nafronyl oxalate salt for samples with various $d e_{2,4}$ ratios ranged from 108 to $112^{\circ} \mathrm{C}$, while the heat of fusion - from 94 to $103 \mathrm{~J} / \mathrm{g}$. No existence of new phase formation was detected within the concentration range investigated. A typical thermogram is presented in supplementary materials in Figure S1.

\subsection{XRPD patterns}

To characterize the crystalline lattice of nafronyl oxalate salt, the XRPD (X-ray powder diffraction) technique was used (section 5.2.7). The samples subjected to the 
measurements were acquired in the same way, as described above in section 2.3. The XRPD spectra are presented in the Figures 4A and 4B.

A)

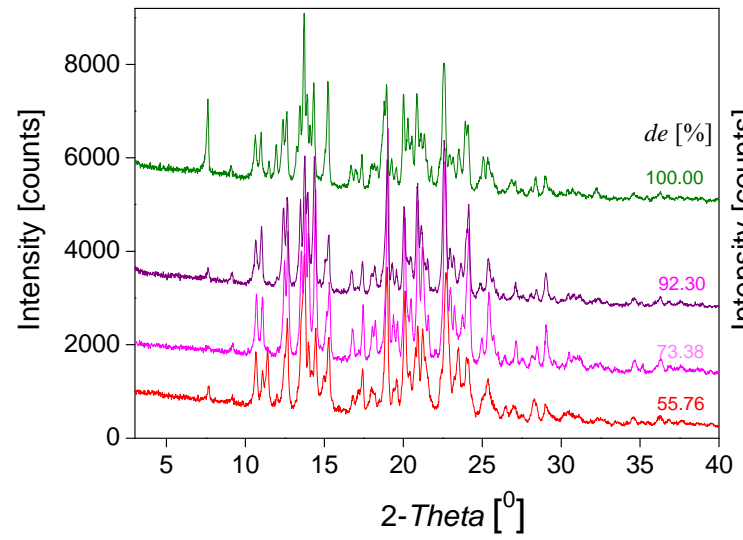

B)

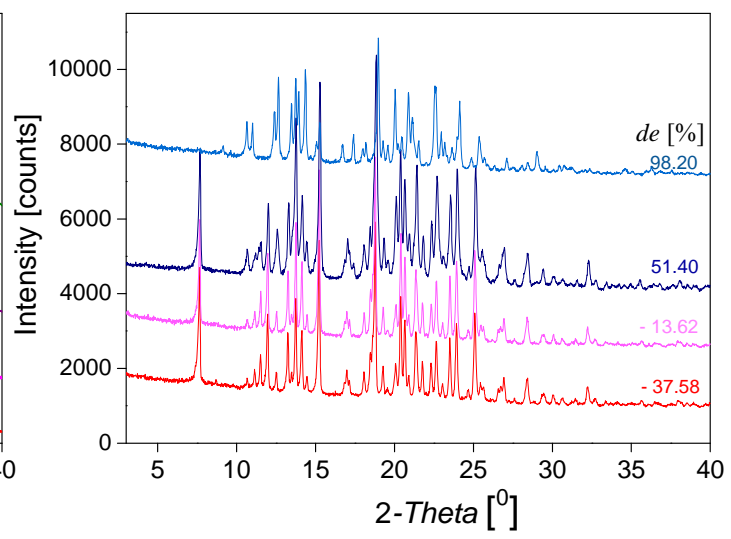

Figure 4. XRPD pattern of stereoisomeric mixture of nafronyl oxalate salt measured at various $d e_{2,4}$ for: A) crystalline phase; B) solid residues of mother liquors.

It can be observed that the patterns obtained for samples with various $d e_{2,4}$ have a similar set of reflexes. A difference in the pattern can be observed only for the extreme excess of the opposite racemate in the mixture. The reported patterns indicate formation of the continuous solid solution, which occurs as a result of mutual substitution of molecules of diastereomers in the crystalline structure. Pure racemates $\mathbf{2 , 4}$ and $\mathbf{1 , 3}$, which are diastereomers of each other can form similar but not identical crystalline lattices. Based on those results, the formation of partial solid solution in the crystalline phase was confirmed, in the agreement to the SLE data reported in section 2.2.

\section{RESULTS AND DISCUSSION}

\subsection{Concept of the separation procedure}

Due to differences in retention properties of stereoisomers on CSP, chromatography can be employed to isolate stereoisomer $\mathbf{4}$ out of the quaternary mixture. The process can be performed batchwise in a single column, or continuously in a multicolumn arrangement. To realize continuous separation, simulated moving bed process (SMB) can be particularly recommended. ${ }^{26-28}$ Though classical SMB process is designed for separation of binary mixtures, it could be easily adopted for the resolution of quaternary mixtures of nafronyl stereoisomers, where the early eluted stereoisomers $\mathbf{1}, \mathbf{2}$, and $\mathbf{3}$ can be considered as a singlecomponent impurity. However, regardless of chromatographic system considered, the efficiency and cost of the process strongly depends on the separation selectivity, which is usually low for multicomponent mixtures. Therefore, we have proposed an alternative 
approach, in which the stereoisomeric mixture was pre-separated prior to the chromatographic process.

Namely, to isolate the target stereoisomer 4 out of the quaternary mixture of stereoisomers three processes were combined; a flowsheet scheme for the separation procedure is depicted in Figure 5. The first process (I) is multistage cross-current crystallization aimed at isolating the racemate $\mathbf{2 , 4}$ that contained the target stereoisomer.

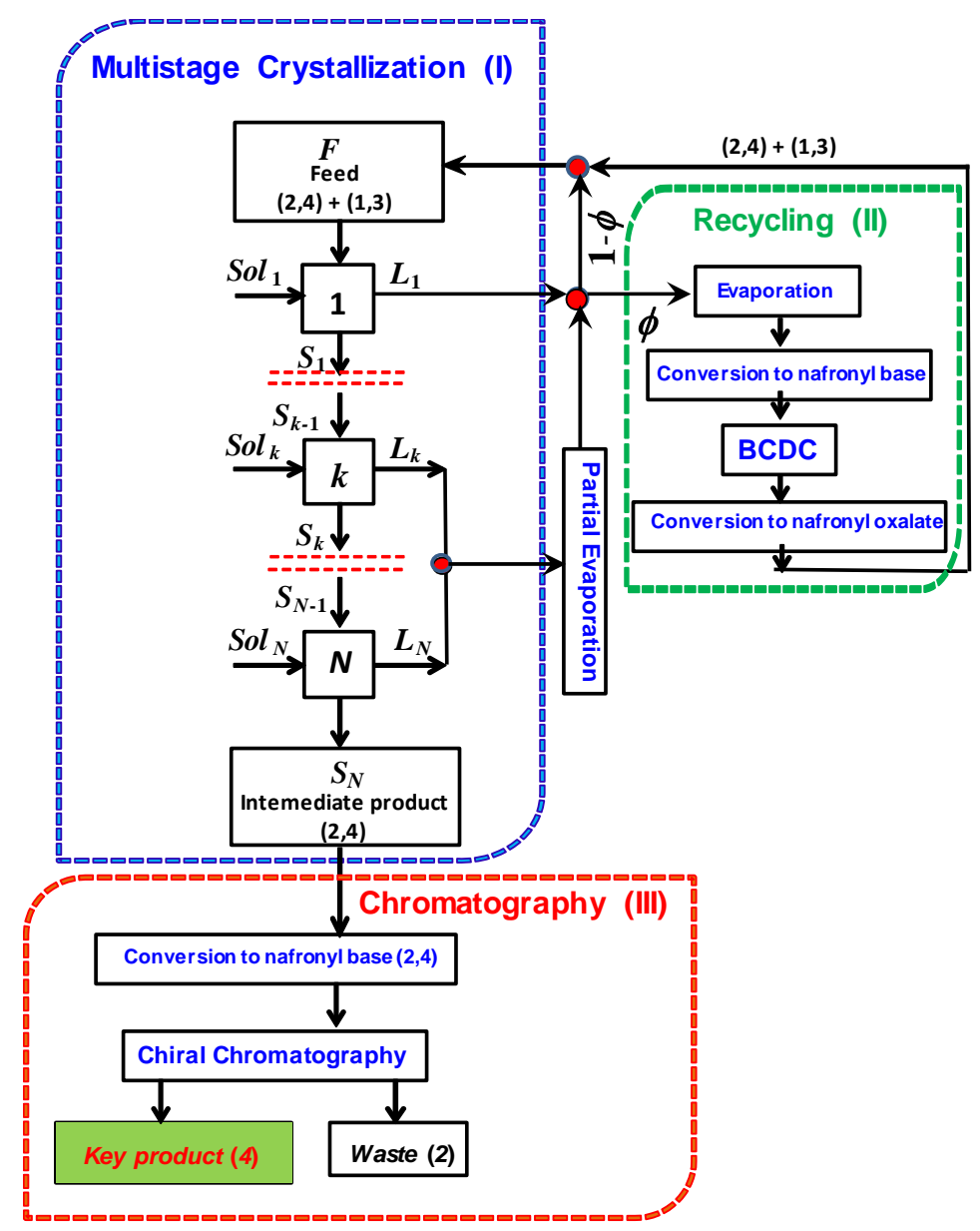

Figure 5. Flowsheet scheme of the separation procedure; $\phi$ - fraction of the mother liquor from the first stage $L_{1}$.

The maximum amount of a single component, which can hypothetically be recovered in the separation process, is determined by the composition of feed mixture. In our study the content of the target component in the raw material was only about $32 \%$. Therefore, to improve the separation recovery, crystallization process was coupled with an additional recycling process (II), which included three operations: a) conversion of stereoisomeric mixture nafronyl oxalate salt to nafronyl free base, $b$ ) base-catalyzed diastereoisomeric conversion (BCDC) of $\alpha$-proton on 2 position in nafronyl molecule in free base form in the presence of sodium hydride as the catalyst, which provided equimolar mixture of nafronyl 
free base stereoisomers, and c) conversion of stereoisomeric mixture of nafronyl free base back to nafronyl oxalate salt (see section 5.3 for the reaction routes). The output solution of the process (II) could be recycled back to the crystallization process by mixing with the mother liquors and feed.

The last process was CSP batch chromatography (III) that was used to isolate the target stereoisomer 4 from the racemate 2,4 . That process can be potentially realized in a continuous system, i.e., in an SMB unit.

\subsection{Multistage cross-current crystallization}

The crystallization process was realized batchwise in the cross-current mode, according to the procedure described in section 5.2.5.

Because preliminary experiments revealed that temperature changes did not influence on the type of the phase behavior, i.e., solid solution was formed in the crystalline phase regardless of the process temperature ranging from $10-40^{\circ} \mathrm{C}$ (data not shown), the process was realized isothermally at $20^{\circ} \mathrm{C}$. A detailed description of the idea behind this process can be found in previous studies. ${ }^{29-31}$ The idea of the process is presented in the upper part of Figure 5 , where a schematic drawing of an $N$-stage cross-current cascade of batch crystallizers is shown. The solid raw material (Feed, $F$ ) is delivered into the first stage of crystallization, $k=1$, where it is mixed with fresh solvent. The solid phase enriched with the target racemate is directly transported into next stages, where the operation is repeated. The crystalline target product is collected at the outlet of the last stage, $N$. A fraction $\phi$ of the mother liquor of stage $k=1, L_{1}$, which is depleted with the target racemate $\left(d e_{2,4}<0 \%\right)$, is subjected to the process (II), and recycled, as described above.

To design the crystallization process, a set of mass balance equations supplemented with the SLE relationship was specified (Appendix). The purity of the product withdrawn from the last stage was set to be minimum $99 \%$. To meet that demand, four stages were required in the cascade, $N=4$. The solution of the set of equations, A1-A9, (Appendix) provided the masses and compositions of all phases, except for the inlet of the first stage, where feed with the known mass and composition was delivered, and the last one, for which the product purity was set.

On the basis of the model predictions the experimental separation was designed and performed according to the procedure described in section 5.2.5. The mass of the solid phases and the mother liquors along with their composition determined using HPLC analysis, and yield of the process are reported in Table 2. 
Table 2. Course of four-stage cross-current crystallization including BCDC: the model predictions (upper rows for each stage) and the experimental results (bottom rows). $Y, Y_{\text {total }}$ defined by Eq. 2 and Eq. 3.

\begin{tabular}{|c|c|c|c|c|c|c|c|c|c|c|c|c|}
\hline \multirow{4}{*}{$\begin{array}{c}\text { Stage } \\
k\end{array}$} & \multirow{2}{*}{\multicolumn{4}{|c|}{ Inlets }} & \multicolumn{8}{|c|}{ Outlets } \\
\hline & & & & & \multicolumn{3}{|c|}{ SOLID } & \multicolumn{4}{|c|}{ LIQUID } & \multirow[b]{2}{*}{$Y$} \\
\hline & $S_{k-1}$ & $S C$ & $d e_{2,4}$ & $x_{2,4, k-1}^{S}$ & $S_{k}$ & $x_{2,4, k}^{S}$ & $d e_{2,4}$ & $L_{k}$ & $\begin{array}{l}X_{1,3, k}^{L} \\
\times 10^{3}\end{array}$ & $\begin{array}{r}x_{2,4, k}^{L} \\
\times 10^{3}\end{array}$ & $d e_{2,4}$ & \\
\hline & {$[\mathrm{g}]$} & {$[\mathrm{g} / \mathrm{g}]$} & {$[\%]$} & {$[\mathrm{g} / \mathrm{g}]$} & {$[\mathrm{g}]$} & {$[\mathrm{g} / \mathrm{g}]$} & {$[\%]$} & {$[\mathrm{g}]$} & {$[\mathrm{g} / \mathrm{g}]$} & {$[\mathrm{g} / \mathrm{g}]$} & {$[\%]$} & {$[-]$} \\
\hline \multirow{2}{*}{1} & 4.690 & 29.44 & 26 & 06300 & 2.5534 & 0.8225 & 65 & 140.23 & 9.1 & 6.1 & -20 & 0.711 \\
\hline & & & & & 2.4675 & 0.8206 & 64 & 139.17 & 8.6 & 6.2 & -16 & 0.685 \\
\hline \multirow{2}{*}{2} & 2.460 & 38.64 & 64 & 0.8206 & 1.5756 & 0.9294 & 86 & 95.95 & 3.4 & 5.8 & 26 & 0.725 \\
\hline & & & & & 1.6329 & 0.9249 & 86 & 89.24 & 3.2 & 5.8 & 29 & 0.748 \\
\hline \multirow{2}{*}{3} & 1.625 & 40.20 & 86 & 0.9249 & 1.1669 & 0.9705 & 94 & 65.78 & 1.2 & 5.6 & 64 & 0.753 \\
\hline & & & & & 1.1860 & 0.9689 & 94 & 68.53 & 1.0 & 5.0 & 67 & 0.764 \\
\hline \multirow{2}{*}{4} & 1.176 & 43.36 & 94 & 0.9689 & 0.8662 & 0.9851 & 98 & 51.30 & 0.4 & 5.6 & 86 & 0.749 \\
\hline & & & & & 0.8335 & 0.9875 & 98 & 47.29 & 0.5 & 5.1 & 82 & 0.723 \\
\hline \multirow{2}{*}{\multicolumn{10}{|c|}{$\begin{array}{l}\text { without recycling of mother liquors, simulated (upper value) and experimental data } \\
\text { (bottom value) }\end{array}$}} & \multirow{2}{*}{\multicolumn{2}{|c|}{$Y_{\text {total }}{ }^{\mathrm{a}}$}} & 0.279 \\
\hline & & & & & & & & & & & & 0.289 \\
\hline \multicolumn{10}{|c|}{$\begin{array}{l}{ }^{\mathrm{b}} \text { with recycling of } 100 \% \mathrm{w} / \mathrm{w} \text { of mother liquors } L \text { from stages } k>1 \text {, and } 36.35 \% \text { for the } \\
\text { stage } k=1 \text { (calculated) }\end{array}$} & \multicolumn{2}{|c|}{$Y_{\text {total }} \mathrm{b}$} & 0.595 \\
\hline \multicolumn{10}{|c|}{$\begin{array}{l}{ }^{c} \text { with recycling of } 100 \% \mathrm{w} / \mathrm{w} \text { of mother liquors } L \text { from stages } k>1 \text { and } 100 \% \text { for the } \\
\text { stage } k=1 \text {, where the fraction } \phi=0.923 \text { of } L_{1} \text { was subjected to BCDC (calculated). Yield } \\
\text { of the whole process (II) comprising BCDC and conversions from oxalate salt to free } \\
\text { base and vice versa, was } 65 \%, d e_{2,4} \text { of the obtained mixture was } 4 \% \text {. }\end{array}$} & \multicolumn{2}{|c|}{$Y_{\text {total }}^{\mathrm{c}}$} & 0.844 \\
\hline
\end{tabular}

Additionally, yield of crystallization coupled with recycling of mother liquors and BCDC was calculated. The fraction, $\phi$ (see Figure 5), of the exhausted mother liquor from the first stage, $L_{1}$, subjected to BCDC, was selected in such a way that $d e_{2,4}$ of the solution obtained after mixing all mother liquors was the same as in feed delivered into the first stage. An improvement of yield could be achieved by optimization of the process. ${ }^{31}$

From Table 2 it is evident that the process is predictable and the desired purity of the product was achieved. The discrepancy between the simulations and the experimental data was caused by experimental errors arising from the operation of phase separation and determination of the SLE relationship.

Because SLE is established after a relatively long time, i.e., about $24 \mathrm{~h}$, the process may start up with repeating twice each crystallization stage. Then, the stored masses of the products obtained in each stage may be subsequently used in four batch crystallizers working in parallel. A further reduction of the process duration is possible, however, the same purity could be achieved at expense of increase in the number of stages. 
The course of the process is also illustrated graphically on the ternary diagram in Figure 3A, where the composition of the initial solutions (mixing points) are marked along with the corresponding tie lines indicating the equilibrium conditions, and on the distribution diagram in Figure 3B, which shows changes in the enrichment of the crystalline phase for each stage. Additionally, the values of the performance indicators for each crystallization stage are depicted in Figure 3C. It can be observed that the phase distribution coefficient reduces at simultaneous enhancement of the solvent consumption with increasing concentration of the racemate $\mathbf{2 , 4}$ in the solution according to SLE data. However, due to synchronization of the operating parameters in the subsequent stages, i.e., the masses and compositions of inlet and outlet streams, based on the solution of the mass balance equations (Appendix, Eqs.A1-A9), the increase in the solvent consumption is much less pronounced compared to that reported for the SLE data. Moreover, yield of each stage remains almost on the same level.

\subsection{Coupling crystallization and base-catalyzed diastereomeric conversion}

To reduce the product losses, a fraction of the exhausted mother liquor of the first stage, $L_{1}$, with $d e_{2,4}<0 \%$ (Table 2) was subjected to the recycling process (II) (see Figure 5). At first, conversion of nafronyl oxalate salt to free base form was performed. Next, the solution was evaporated and subjected to BCDC, which provided an equimolar stereoisomeric mixture that could be recycled to crystallization. BCDC was performed according to the procedure described in section 5.3.2 for several quaternary stereoisomeric mixtures of nafronyl free base differing in $d e_{2,4}$. In all cases equimolar mixture 1:1:1:1 of four stereoisomers was obtained, i.e., $d e_{2,4}=0$, regardless of the initial $d e_{2,4}$ of the sample. The post-BCDC solution was converted back into the oxalate form, $d e_{2,4}$ of the obtained mixture was $4 \%$. Yield of the whole process (II) (with respect to the theoretical value resulting from the reaction stoichiometry) was $65 \%$.

The coupling of crystallization with recycling of the mother liquors and BCDC can benefit in significant improvement of yield for the target racemate $\left(Y_{\text {total }}[\%]=84 \%\right)$ compared to the crystallization process coupled solely with recycling of the exhausted mother liquors $(60 \%)$ and to single crystallization without recycling (29\%) (Table 2, Eq. 3).

\subsection{CSP batch chromatography}

The pure racemate $\mathbf{2 , 4}$ obtained from the crystallization process was further processed by chiral chromatography (Figure 5) in a medium pressure semi-preparative system (section 5.2.2) to isolate the desired stereoisomer 4. The efficiency of chromatographic separation for 
the binary mixture of enantiomers was much higher compared to that for the quaternary mixture of all stereoisomers. The latter required a highly efficient column HPLC working in a high pressure system at low column load, whereas the former could be realized in a medium pressure semi-preparative system, under high mass overloading conditions. Therefore, coupling of diastereoisomeric crystallization, aimed at isolate the target racemate, with chiral chromatography, designed for providing the pure stereoisomer, can be expected to improve economics of the whole separation process.

To increase the column load, the racemate $\mathbf{2 , 4}$ was converted from oxalate salt form to nafronyl free base (section 5.3.1). The maximum solubility of nafronyl free base in the mobile phase is about $50 \mathrm{~g} / \mathrm{L}$, whereas nafronyl oxalate salt is scarcely soluble in the mobile phase used for chromatography.

A typical course of the chromatographic elution for racemate 2,4 is shown in Figure 6. The productivity of the separation calculated as the amount of pure product collected at the column outlet (stereoisomer 4 with HPLC area purity above 99\%) per the duration of single chromatographic cycle and per the column volume was $1.27 \mathrm{~g} \mathrm{~h}^{-1} \mathrm{~L}^{-1}$. Yield for the target compound in case of the touching band separation showed in Figure 6 was 99\%. Therefore, total yield loss for the whole process was determined by crystallization and recycling process including BCDC. Because BCDC was directed to $\alpha$-proton on 2 position in nafronyl molecule, the stereoisomer 2 wasted in the chromatographic separation could not be recycled - BCDC for that stereoisomer provided undesirable pair of diastereomers, 2,3.

The chromatographic process presented in this study, can be easily scaled up according to a general rule expressed by two equations of equality of the linear velocities, $w$, in a small column, $a$, and a large column, $l:^{32}$

$$
w_{l}=w_{a}
$$

and equality of the ratio of the columns lengths, $L$, and the squared ratio of the bed particle diameters, $d_{p}$ :

$$
\frac{L_{l}}{L_{a}}=\left(\frac{d_{p, l}}{d_{p, a}}\right)^{2}
$$

The ratio of loading volumes $\left(V_{\text {load }}\right)$ should be equal to the ratio of column volumes $\left(V_{\text {column }}\right)$ :

$$
\frac{V_{\text {load }, l}}{L_{\text {load }, a}}=\frac{V_{\text {column }, l}}{L_{\text {column }, a}}
$$


If the equalities given by Eqs. (6-8) are fulfilled, the separation productivity for both columns should be the same.

The process performance may be further optimized with regard to productivity of the process realized in batch as well as continuous mode (SMB). This requires determining basic parameters of the chromatographic process, including adsorption isotherm coefficients and the column efficiency, which can be easily done using standard experimental and numerical methods (see e.g., ${ }^{32,33}$ ).

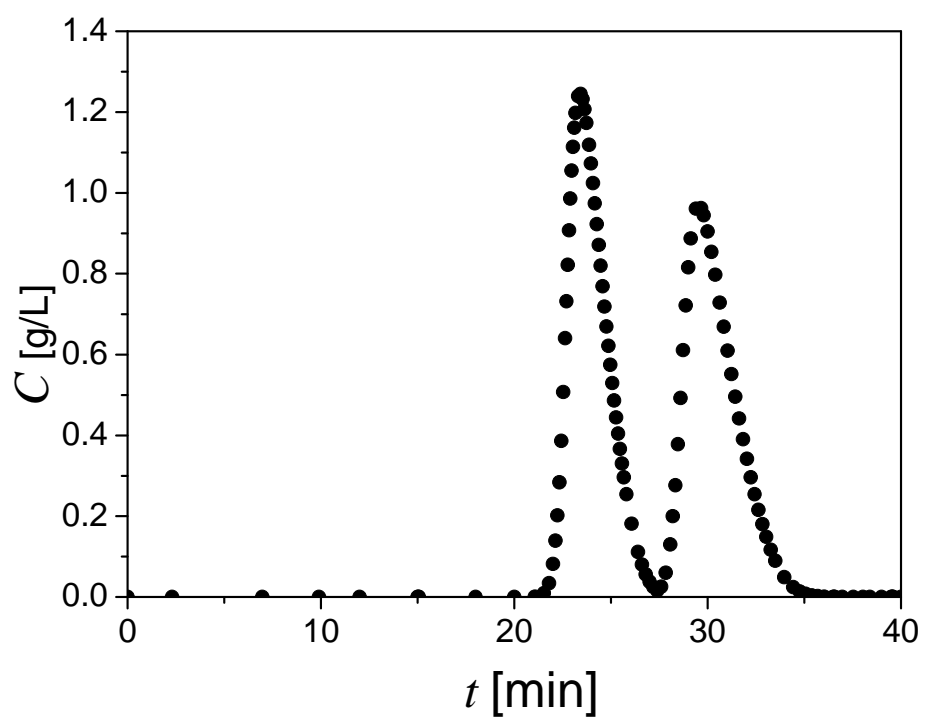

Figure 6. Illustration of separation of the racemate 2,4 in the medium pressure chromatographic system; the concentration of racemate 2,4 in the sample $-30 \mathrm{~g} / \mathrm{L}$, injection volume - $0.1 \mathrm{~mL}$, flowrate $0.5 \mathrm{~mL} / \mathrm{min}$.

\section{CONCLUSIONS}

In this study we have developed a procedure for the isolation of the most biologically active compound out of the quaternary mixture of nafronyl oxalate salt stereoisomers. The research objective was a representative of compounds possessing two chiral centres, which can occur in the form of four stereoisomers. The procedure consisted of three processes: multistage cross-current crystallization of stereoisomeric mixtures in acetone, base-catalysed diasteromeric conversion (BCDC) with sodium hydride as the catalyst, and chiral chromatography. The crystallization process allowed isolation of the racemate that contained the target stereoisomer. The process design was preceded by measurements of the solid-liquid equilibrium of stereoisomers of nafronyl oxalate salt in acetone. The shape of the ternary phase diagram indicated that the stereoisomeric mixture exhibited the solid solution in the crystalline phase with the minimum solubility for the pure target racemate, and that the excess of the target racemate could be increased in the crystalline phase. Based on the results of the 
SLE measurements, the condition for four-stage crystallization was selected. The separation was experimentally realized; yield and purity of the product obtained were in good agreement with the mathematical predictions.

To improve the process yield, the mother liquor depleted with the target racemate was subjected to BCDC. The combination of cross-current crystallization and BCDC could benefit in the improvement of the separation yield for the target racemate from about 29 to $84 \%$.

Further, the purified racemate was resolved into its pure enantiomers using CSP batch chromatography to obtain the most active stereoisomer. Separation of the binary mixture could be performed easily using the available CSP at a high purity, yield and under conditions of a high column load.

The suggested concept of the coupled process might potentially be adopted in separations of stereoisomers of other compounds with two stereogenic centers.

\section{EXPERIMENTAL SECTION}

\subsection{Chemicals}

The stereoisomeric mixture of nafronyl oxalate salt was purchased from Santa-Cruz Biotechnology (Santa Cruz Biotechnology, Santa Cruz, USA). The diasteromeric enrichment of the target racemate, $d e_{2,4}$, in the purchased material was $26 \%$. To accomplish the crystallization and chemical reactions the following chemicals were used: acetone, $\mathrm{Pu} \geq$ 99.8\% (VWR Prolabo); dichloromethane, $\mathrm{Pu} \geq 99.8 \%$ (VWR Prolabo); propan-2-ol, $\mathrm{Pu} \geq$ 99.9\% (POCH); $1 \mathrm{M}$ sodium hydroxide, $\mathrm{Pu} \geq 99 \%$ (Merck); magnesium sulfate anhydrous, $\mathrm{Pu}$ $\geq 99 \%$ (Sigma-Aldrich); sodium hydride 60\% w/w dispersion in mineral oil (Sigma-Aldrich) and oxalic acid dihydrate, $\mathrm{Pu} \geq 99.5 \%$ (Chempur). Water was filtered using a Milli-Q unit equipped with $0.22 \mu \mathrm{m}$ membrane (Millipore, Bedford, USA). For the chromatographic separations n-hexane, $\mathrm{Pu} \geq 99 \%$ and propan-2-ol, $\mathrm{Pu} \geq 99.9 \%$, with HPLC-grade (VWR Prolabo) and diethylamine, $\mathrm{Pu} \geq 99.5 \%$ (Sigma-Aldrich), were used.

\subsection{Procedures}

\subsubsection{HPLC analysis}

The HPLC analysis was performed with Agilent 1200 Series System (Agilent Technologies, Santa Clara, USA). The HPLC device consisted of a high pressure quaternary pump with a vacuum degasser, autosampler, thermostatted column compartment, diode array detector and advanced laser polarimeter (PDR-Chiral Inc., Lake Park, USA). The 
ChemStation software was used for the analysis of chromatographic profiles. The system was equipped with an analytical column CHIRALPAK IC purchased from Chiral Technologies (Chiral Technologies Europe, Illkirch Cedex, France). The chiral stationary phase consisted of cellulose tris(3,5-dichlorophenylcarbamate) as a chiral selector, which was immobilized onto a silica matrix. The HPLC system is characterized in Table 3.

All samples destined for the HPLC measurements were dissolved in propan-2-ol. The concentration of samples acquired from the crystalline phases was $1 \mathrm{~g} / \mathrm{L}$. The samples of mother liquors were acquired with the amount of $200 \mu \mathrm{L}$ and diluted 50 times.

Their concentrations were determined from the peak area using the detector calibration factor.

Table 3. Characterization of chromatographic systems

\begin{tabular}{|c|c|c|}
\hline Column dimensions $[\mathrm{mm}]:$ & \multicolumn{2}{|c|}{ CHIRALPAK-IC } \\
\hline Stationary phase: & \multicolumn{2}{|c|}{25} \\
\hline Mobile phase: & n-hexane/propan-2-ol/diethylamine 90/10/0.1 (v/v) \\
\hline$t\left[{ }^{\circ} \mathrm{C}\right]:$ & $\begin{array}{c}\text { Migh pressure } \\
\text { (analytical) }\end{array}$ & $\begin{array}{c}\text { Medium pressure } \\
\text { (semi-preparative) }\end{array}$ \\
\hline DAD Detection $[\mathrm{nm}]:$ & 226 & 320 \\
\hline Particle size $[\mu \mathrm{m}]$ & 5 & 20 \\
\hline Flow rate $[\mathrm{mL} / \mathrm{min}]:$ & 1 & 100 \\
\hline Injection $[\mu \mathrm{L}]:$ & 2 & 0.5 \\
\hline
\end{tabular}

\subsubsection{Medium pressure chromatography}

The medium pressure chromatographic resolution of the racemate 2,4 was performed using the same HPLC system, as described above in section 5.2.1. However, in this case the system was equipped with the CHIRALPAK IC column packed with larger particles compared to the high pressure analytical chromatography. That stationary phase is designed for semi-preparative or preparative separations. The conditions for the separation are presented in Table 3.

The feed mixture that contained the racemate 2,4 was prepared by dissolving nafronyl free base in the mobile phase to obtain the total concentration of $30 \mathrm{~g} / \mathrm{L}$.

\subsubsection{Isolation of the racemate 2,4 from the raw material by multistage crystallization}

To provide a suitable amount of the pure racemate $\mathbf{2 , 4}$, necessary to perform the SLE measurements (section 5.2.4), the raw stereoisomeric mixture of nafronyl oxalate salt $\left(d e_{2,4}=26 \%\right)$ was subjected to multistage crystallization. Based on the literature data acetone was selected as a solvent for crystallization. ${ }^{23}$ The crystallization process was realized in six 
stages. In the first stage $10 \mathrm{~g}$ nafronyl oxalate salt from the raw material and $200 \mathrm{~mL}$ acetone were placed into a glass double-walled thermostatted vessel with the hermetic lid, equipped with a magnetic stirrer. The batch was heated up to $50^{\circ} \mathrm{C}$ in a water bath until entire dissolution. Subsequently, the solution was stirred at temperature $20^{\circ} \mathrm{C}$, frequency of rotation $300 \mathrm{rpm}$ for $24 \mathrm{~h}$, in order to establish the solid-liquid equilibrium. Next, the mixture was left in a thermostat (Thermostat Lauda Ecoline RE-104, Germany) for $20 \mathrm{~min}$. without stirring, to allow separation of phases. Samples of the mother liquor were acquired using a syringe with a filter having anhydrous pores $0.45 \mu \mathrm{m}$. Then, the solid phase was separated out of the mother liquor using vacuum filtration and dried in a vacuum dryer at temperature $50^{\circ} \mathrm{C}$ and pressure $0.4 \mathrm{bar}$, for $24 \mathrm{~h}$. Subsequently, the acquired samples were prepared for the HPLC analysis according to procedure described above in section 5.2.1. The enriched crystalline phase was mixed with pure acetone and subjected again to crystallization for further enrichment. The amount of acetone was adjusted according to the solubility of the stereoisomeric mixture. The procedure was repeated six times. The $d e_{2,4}$ of the crystalline phase obtained in the sixth stage was about $99 \%$ and total yield $27 \%$.

\subsubsection{Determination of SLE for stereoisomeric mixtures of nafronyl oxalate salt}

To quantify SLE, several samples of the crystalline phase were prepared by mixing the raw material with pure racemate 2,4 to obtain various $d e_{2,4}$ ratios, ranged from $26 \%$ (raw material) to $99 \%$ (pure racemate 2,4 ). The solid mixture was suspended in acetone in closed glass vials. The volume of solvent was adjusted for each sample based on the sample solubility to obtain supersaturated solutions. All samples were heated up to $50^{\circ} \mathrm{C}$ in water bath for complete dissolution. Subsequently, the vials were placed into a water-jacketed vessel integrated with the thermostat and the solutions were electromagnetically stirred at temperature $20^{\circ} \mathrm{C}$, frequency of rotation $300 \mathrm{rpm}$, for $24 \mathrm{~h}$, to establish solid-liquid equilibrium. Then, the samples were centrifuged at $20^{\circ} \mathrm{C}, 1000 \mathrm{rpm}$, for $2 \mathrm{~min}$.

Samples of the mother liquors and crystalline phases were acquired in the same manner as described above in section 5.2.3 and subjected to the HPLC analysis (section 5.2.1).

\subsubsection{Four-stage cross-current crystallization to isolate the racemate 2,4 from quaternary mixture}

The crystallization process was realized batchwise according to the scheme presented in Figure 5. In each stage crystallization was performed in a closed vessel, which was 
supplied with feed and acetone. The solution was heated up to $50^{\circ} \mathrm{C}$ in water bath until complete dissolution. this accelerated the kinetic rate of crystallization process. Afterwards, the solution was rapidly cooled in the thermostat at $20^{\circ} \mathrm{C}$ (according to the same procedure as described in section 5.2.3). After establishing SLE the samples of mother liquors and crystalline phases were acquired for the HPLC analysis (section 5.2.1). The crystalline phases were weighted and used to prepare feed for a subsequent stage. The procedure was repeated four times. The crystalline phase obtained from the last stage was the final product of the crystallization process. The mother liquor depleted with the target racemate was subjected to BCDC

\subsubsection{DSC Analysis}

Thermal analysis was performed by DSC using Setaram DSC131 instrument (Setaram, France). Samples with 7-13 mg acquired from the crystalline phases or the residues of the mother liquors after evaporation and drying were closed in $30 \mu \mathrm{L} \mathrm{Al-crucibles} \mathrm{under} \mathrm{a} \mathrm{helium}$ atmosphere. The temperature program was set to heat up the samples from 25 to $140^{\circ} \mathrm{C}$ at a constant rate of $5 \mathrm{~K} / \mathrm{min}$.

\subsubsection{XRPD Analysis}

The samples of crystal powder from crystalline phases and the residues of mother liquors were subjected to the XRPD analysis using PANalytical X'Pert Pro diffractometer (PANalytical GmbH, Germany) with an $\mathrm{X}^{\prime}$ Celerator detector. The radiation source was $\mathrm{Cu}$ $\mathrm{K} \alpha$. Samples were measured on Si holders and scanned in a 2-Theta range of 3 to $40^{\circ}$ with a step size of $0.017^{\circ}$ and counting time of $50 \mathrm{~s}$ for each step.

\subsection{Reaction routes}

\subsubsection{Conversion of nafronyl oxalate salt into nafronyl free base}

For conversion of nafronyl oxalate salt to nafronyl free base a similar procedure was used as described by Kempen. ${ }^{20}$ The reaction scheme is given in Figure 7A.

A $2 \mathrm{~g}$ of nafronyl oxalate salt $(\mathrm{M}=473.56 \mathrm{~g} / \mathrm{mol})$ was dissolved in $20 \mathrm{~mL}$ dichloromethane and $20 \mathrm{~mL}$ water. Next, $6 \mathrm{~mL}$ of $1 \mathrm{M}$ sodium hydroxide solution was slowly added. The mixture was intensively stirred at ambient temperature for $30 \mathrm{~min}$, and then the aqueous and organic phases were separated. The aqueous phase was extracted with dichloromethane, while the combined organic phase was washed several times using water until neutral $\mathrm{pH}$ of the solution was reached. The organic phase was dried with anhydrous magnesium sulfate for $1 \mathrm{~h}$. After filtrating out the drying agent, the filtrate was concentrated 
using an evaporator. The solvent residue was removed by a vacuum drier at temperature $50^{\circ} \mathrm{C}$, pressure $0.4 \mathrm{bar}$, for $24 \mathrm{~h}$. Nafronyl free base $(\mathrm{M}=383.52 \mathrm{~g} / \mathrm{mol})$ was obtained with a gold-colored thickened consistency. The reaction yield was $90 \%$ (with respect to the theoretical value resulting from the reaction stoichiometry).

\subsubsection{Base-catalyzed diastereoisomeric conversion (BCDC)}

BCDC was a base-catalyzed reaction in melt, where as a catalyst sodium hydride was used. The reaction scheme is given in Figure 7B.

A)

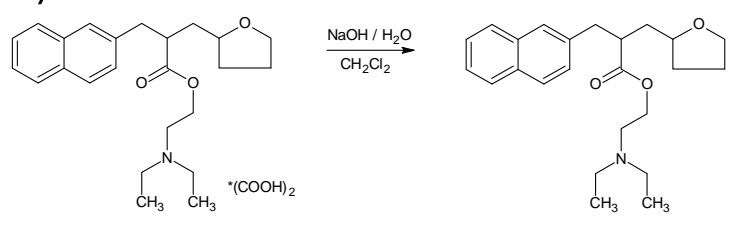

B)

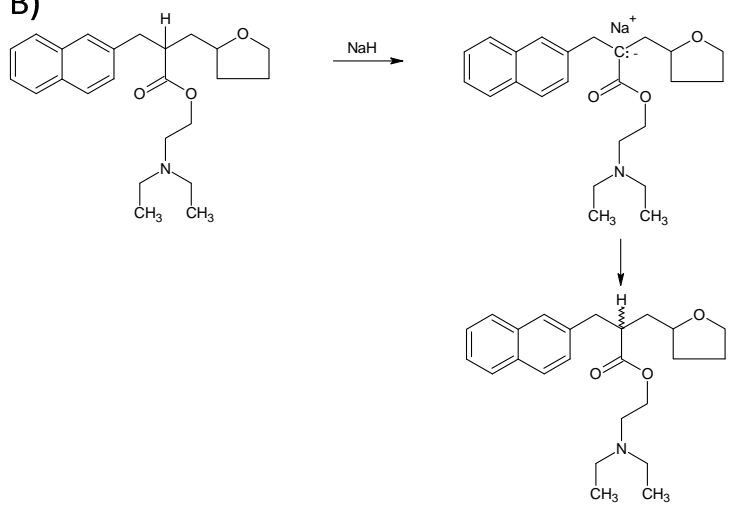

C)

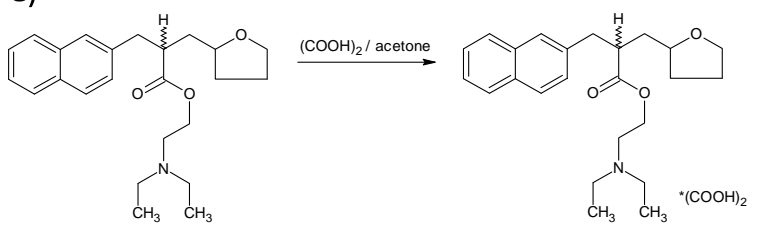

Figure 7. A) Conversion of nafronyl oxalate salt to nafronyl free base; B) racemic transformation in the melt; C) conversion of nafronyl free to nafronyl oxalate salt.

A $0.69 \mathrm{~g}(1,77 \mathrm{mmol})$ nafronyl free base and $0.079 \mathrm{~g}(\sim 2.01 \mathrm{mmol})$ sodium hydride in the form of a $60 \% \mathrm{w} / \mathrm{w}$ suspension in mineral oil, were placed into to a tightly closed flask. Then, dry nitrogen was blown through the mixture for 15 minutes. After that, the flask was sealed and heated up to $90^{\circ} \mathrm{C}$ for 3 hours. Next, the reaction mixture was cooled down into room temperature and treated with $1 \mathrm{ml}$ of propan-2-ol. The residue was stirred until solid was dissolved, and then, $10 \mathrm{~mL}$ of water was added. The mixture was placed into a separator funnel and extracted with $20 \mathrm{ml}$ of dichloromethane. The organic layer was separated out and dried over anhydrous magnesium sulphate. Next, the solvent was evaporated to obtain $0.62 \mathrm{~g}$ 
$(\sim 1.59 \mathrm{mmol})$ of equimolar mixture of nafronyl free base stereoisomers with yield $90 \%$, and HPLC area purity $98 \%$.

\subsubsection{Conversion of nafronyl free base to nafronyl oxalate salt}

For conversion of nafronyl free base to nafronyl oxalate salt a similar procedure was used as described by Hao et al. ${ }^{21}$ The reaction scheme is given in Figure 7C. A $0.15 \mathrm{~g}$ of oxalic acid dihydrate was added to a solution of $0.46 \mathrm{~g}(1.20 \mathrm{mmol})$ nafronyl free base in 3 $\mathrm{mL}$ of acetone. The reaction mixture was heated under reflux for $1 \mathrm{~h}$ and then cooled down to the room temperature. Under those conditions spontaneous crystallization occurred after 1 hour. The crystalline phase was equilibrated with the solution for 24 hours, then filtrated out. $0.46 \mathrm{~g}(\sim 0.97 \mathrm{mmol})$ of nafronyl oxalate salt was obtained with yield $80 \%$, and HPLC area purity $99 \%$.

\subsection{Spectral data}

2-(diethylamino)ethyl 3-(naphthalen-1-yl)-2-((tetrahydrofuran-2-yl)methyl)propanoate oxalate - the product of four-stage crystallization (the target racemate, 2,4), Figures S2a-2c in Supplementary Materials.

${ }^{1}$ H NMR: $\delta \mathrm{H}\left(500 \mathrm{MHz} ; \mathrm{CDCl}_{3} ; \mathrm{Me}_{4} \mathrm{Si}\right)$ 0,85-1,13 (m, 6H); 1,44-1,56 (m, 1H); 1,77-1,96 (m, $3 \mathrm{H}) ; 1,97-2,08$ (m, 2H); 2,28-2,84 (m, 5H); 2,98-3,10 (m, 2H); 3,18-3,27 (m, 1H); 3,37-3,44 $(\mathrm{m}, 1 \mathrm{H}) ; 3,66-3,72(\mathrm{~m}, 1 \mathrm{H}) ; 3,76-3,84(\mathrm{~m}, 1 \mathrm{H}) ; 3,92-4,00(\mathrm{~m}, 1 \mathrm{H}) ; 4,14-4,18(\mathrm{~m}, 2 \mathrm{H}) ; 7,28$ $(\mathrm{d}, \mathrm{J}=6.4 \mathrm{~Hz} ; 1 \mathrm{H}) ; 7,38(\mathrm{t}, J=8.25 \mathrm{~Hz}, 1 \mathrm{H}) ; 7,48-7,51(\mathrm{~m}, 1 \mathrm{H}) ; 7,54-7,56(\mathrm{~m}, 1 \mathrm{H}) ; 7,74(\mathrm{~d}$, $J=8,25 \mathrm{~Hz}, 1 \mathrm{H}) ; 7,85(\mathrm{~d}, J=7,65 \mathrm{~Hz}, 1 \mathrm{H}) ; 8,01(\mathrm{~d}, J=8,55 \mathrm{~Hz}, 1 \mathrm{H}) .{ }^{\mathbf{1 3}} \mathbf{C}$ NMR: $\delta \mathrm{H}(500 \mathrm{MHz}$; $\left.\mathrm{CDCl}_{3} ; \mathrm{Me}_{4} \mathrm{Si}\right) 8,56 ; 8,56 ; 25,50 ; 31,67 ; 36,30 ; 38,64 ; 45,15 ; 46,94 ; 46,94 ; 48,71 ; 57,92$; 67,$57 ; 77,10 ; 123,57 ; 125,48 ; 125,89 ; 126,38 ; 127,36 ; 127,50 ; 128,84 ; 131,75 ; 133,85$; 135,$02 ; 163,07 ; 163,07 ; 175,04$.

2-(diethylamino)ethyl 3-(naphthalen-1-yl)-2-((tetrahydrofuran-2-yl)methyl)propanoate nafronyl free base before BCDC, Figures S3a-3c in Supplementary Materials.

${ }^{1} \mathbf{H}$ NMR: $\delta \mathrm{H}\left(500 \mathrm{MHz} ; \mathrm{CDCl}_{3} ; \mathrm{Me}_{4} \mathrm{Si}\right) \quad 0,95$ (t, $\left.J=7.05 \mathrm{~Hz}, 6 \mathrm{H}\right) ; 1,35-1,44(\mathrm{~m}, 1 \mathrm{H}) ; 1,66-$ $1,72(\mathrm{~m}, 1 \mathrm{H}) ; 1,77-1,99(\mathrm{~m}, 3 \mathrm{H}) ; 2,02-2,10(\mathrm{~m}, 1 \mathrm{H}) ; 2,38-2,52(\mathrm{~m}, 5 \mathrm{H}) ; 2,54-2,60(\mathrm{~m}, 1 \mathrm{H})$; 2,94-3,03 (m, 1H); 3,26-3,39 (m, 2H); 3,65-3,72 (m, 1H); 3,77-3,85 (m, 1H); 3,85-3,94 (m, $1 \mathrm{H}) ; 4,00-4,10(\mathrm{~m}, 2 \mathrm{H}) ; 7,35(\mathrm{~d}, J=6,45 \mathrm{~Hz}, 1 \mathrm{H}) ; 7,36(\mathrm{t}, \mathrm{J}=7.95,1 \mathrm{H}) ; 7,45-7,48(\mathrm{~m}, 1 \mathrm{H})$; $7,5-7,56(\mathrm{~m}, 1 \mathrm{H}) ; 7,72(\mathrm{~d}, J=7,95 \mathrm{~Hz}, 1 \mathrm{H}) ; 7,83(\mathrm{~d}, J=8.55 \mathrm{~Hz}, 1 \mathrm{H}) ; 8,05(\mathrm{~d}, J=8,50 \mathrm{~Hz}$, $1 \mathrm{H}) .{ }^{13} \mathrm{C}$ NMR: $\delta \mathrm{H}\left(500 \mathrm{MHz} ; \mathrm{CDCl}_{3} ; \mathrm{Me}_{4} \mathrm{Si}\right) 11,35 ; 11,35 ; 25,54 ; 31,50 ; 35,96 ; 38,13$; 44,$61 ; 47,34 ; 47,34 ; 50,44 ; 61,66 ; 67,55 ; 77,03 ; 123,69 ; 125,35 ; 125,59 ; 126,09 ; 127,29$; 127,$31 ; 128,79 ; 131,90 ; 133,90 ; 135,18 ; 175,56$.

2-(diethylamino)ethyl3-(naphthalen-1-yl)-2-((tetrahydrofuran-2-yl)methyl)propanoate nafronyl free base after BCDC, Figures S4a-4c in Supplementary Materials.

${ }^{1}$ H NMR: $\delta \mathrm{H}\left(500 \mathrm{MHz} ; \mathrm{CDCl}_{3} ; \mathrm{Me}_{4} \mathrm{Si}\right)$ 0.91-1.03 (m, 6H); 1.34-1.48 (m, 1H); 1.65-1.91 (m, $3 \mathrm{H}) ; 1.92-2.11(\mathrm{~m}, 2 \mathrm{H}) ; 2.34-2.67(\mathrm{~m}, 6 \mathrm{H}) ; 2.95-3.16(\mathrm{~m}, 1 \mathrm{H}) ; 3.25-3.41(\mathrm{~m}, 2 \mathrm{H}) ; 3.61-3.72$ $(\mathrm{m}, 1 \mathrm{H}) ; 3.74-3.93(\mathrm{~m}, 2 \mathrm{H}) ; 3.97-4.12(\mathrm{~m}, 2 \mathrm{H}) ; 7.35(\mathrm{~d}, J=6.45 \mathrm{~Hz}, 1 \mathrm{H}) ; 7.36$ $(\mathrm{t}, J=7,6 \mathrm{~Hz}, 1 \mathrm{H}) ; 7.44-7.50(\mathrm{~m}, 1 \mathrm{H}) ; 7.50-7.56(\mathrm{~m}, 1 \mathrm{H}) ; 7,72(\mathrm{~d}, J=8,25 \mathrm{~Hz}, 1 \mathrm{H}) ; 7.84$ 
$(\mathrm{d}, J=7,90 \mathrm{~Hz}, 1 \mathrm{H}) ; 8.00-8.10(\mathrm{~m}, 1 \mathrm{H}) .{ }^{13} \mathrm{C}$ NMR: $\delta \mathrm{H}\left(500 \mathrm{MHz} ; \mathrm{CDCl}_{3} ; \mathrm{Me}_{4} \mathrm{Si}\right)$ 11.33; $11.33 ; 25.53 ; 31.50 ; 36.47 ; 38.26 ; 44.36 ; 47.33 ; 47.33 ; 50.50 ; 61.63 ; 67.54 ; 77.03 ; 123.71$; $125.35 ; 125.57 ; 126.08 ; 127.13 ; 127.27 ; 128.79 ; 131.94 ; 133.88 ; 135.19 ; 175.54$.

2-(diethylamino)ethyl 3-(naphthalen-1-yl)-2-((tetrahydrofuran-2-yl)methyl)propanoate oxalate - nafronyl oxalate salt after conversion form nafronyl free base obtained after BCDC, Figures S5a-5c in Supplementary Materials.

${ }^{1}$ H NMR: $\delta \mathrm{H}\left(500 \mathrm{MHz} ; \mathrm{CDCl}_{3} ; \mathrm{Me}_{4} \mathrm{Si}\right) 0.96(\mathrm{~s}, 6 \mathrm{H}) ; 1.45-1.54(\mathrm{~m}, 1 \mathrm{H}) ; 1.77-1.96(\mathrm{~m}, 3 \mathrm{H})$; 1.97-2.17 (m, 2H); 2.29-2.85 (m, 5H); 2.97-3.18 (m, 2H); 3.19-3.28 (m, 1H); 3.37-3.44 (m, $1 \mathrm{H})$; 3.65-3.74 (m, 1H); 3.76-4.00 (m, 2H); 4.10-4.19 (m, 2H); 7.28-7.32 (m, 1H); 7.36-7.41 $(\mathrm{m}, 1 \mathrm{H}) ; 7.47-7.52(\mathrm{~m}, 1 \mathrm{H}) ; 7.52-7.58(\mathrm{~m}, 1 \mathrm{H}) ; 7.74(\mathrm{~d}, J=8.25 \mathrm{~Hz}, 1 \mathrm{H}) ; 7.85(\mathrm{~d}, J=8,25$, $1 \mathrm{H}) ; 7.97-8.05(\mathrm{~m}, 1 \mathrm{H}) .{ }^{13} \mathrm{C}$ NMR: $\delta \mathrm{H}\left(500 \mathrm{MHz} ; \mathrm{CDCl}_{3} ; \mathrm{Me}_{4} \mathrm{Si}\right) 8.59 ; 8.59 ; 25.57 ; 31.68$; $36.34 ; 38.69 ; 44.33 ; 46.95 ; 46.95 ; 48.85 ; 57.84 ; 67.76 ; 77.06 ; 123.58 ; 125.51 ; 125.93$; $126.41 ; 127.39 ; 127.51 ; 128.84 ; 131.76 ; 133.86 ; 135.02 ; 162.87 ; 162.87 ; 174.98$.

\section{ASSOCIATED CONTENT}

Supporting Information: DSC thermogram, ${ }^{1} \mathrm{H},{ }^{13} \mathrm{C}$ NMR spectral data.

\section{AUTHOR INFORMATION}

Corresponding Authors

*Telephone: +48 178621745; e-mail: maciejb@uniw.rzeszow.pl (M. Balawejder)

*Telephone: +48 178651853; e-mail: dorota.antos@.prz.edu.pl (D. Antos)

\section{Notes}

The authors declare no competing financial interest.

\section{ACKNOWLEDGEMENTS}

Financial support of this work by National Science Center (project UMO2013/08/M/ST8/00982) is gratefully acknowledged.

\section{REFERENCES}

(1) Tucker G.; Lancet, 2000, 355, 1085-1087.

(2) Caner H, Groner E, Levy L.; Drug Discovery Today, 2004, 9, 105-110.

(3) Shimazawa R., Nagai N., Toyoshima S., Okuda H.; J. Health Sci., 2008, 54, 23-29.

(4) Nerkar A, Lade K, Gadhave N, Sawant S.; J. Pharm. Res., 2011, 4, 1300-1303.

(5) N. Maier, P. Franco, W. Lindner; J. Chromatogr. A, 2001, 906, 3-33.

(6) Francotte E., Lindner W., Chirality in Drug Res., Wiley-VCH, Weinheim, 2006.

(7) Riehl J., Mirror - Image Asymmetry an Introduction to the Origin and Consequences of Chirality, Wiley, Canada, 2010.

(8) Karamertzanis P. G., Anandamanoharan P. R., Fernandes P., Cains P.W., Vickers M., Tocher D.A., Florence A.J., Price S.L., J. Phys. Chem. B, 2007, 111, 5326-5336. 
(9) Li. Z., Grant D., J. Pharm.Sci., 1997, 86, 1439-1447.

(10) Gu C.-H., Grant D. J. W., Physical Properties and Crystal Structures of Chiral Drugs; In Handbook of Experimental Pharmacology: Stereochemical Aspects of Drug Action and Disposition; Springer, Berlin, 2003.

(11) Rekoske J. E., AIChE J. 2001, 47, 275-299.

(12) Collet A., Enantiomer. 1999, 4, 157-172.

(13) Müller S., Afraz M. C., Gelder R., Ariaans G. J. A., Kaptein B., Broxterman Q. B., Bruggink A., Eur. J. Org. Chem., 2005, 6, 1082-1096.

(14) Ferreira F.C., Ghazali N.F., Cocchini U., Livingston A.G., Tetrahedron, 2006, 17, 13371348.

(15) Lorenz H., Sheehan P., Seidel-Morgenstern A., J. Chromatogr. A, 2001, 908, 201-214.

(16) Leusen F., Noordik J., Karfunkel H., Tetrahedron, 1993, 49, 5377.

(17) Brands K.M.J., Davies A. J., Chem. Rev. 2006, 106, 2711-2733.

(18) Barradell L. B., Broqden R. N., Drugs \& Aging, 1996, 8, 299.

(19) Goldsmith D. R., Wellington K., Drugs \& Aging, 2005, 22, 967.

(20) Kempen H. J., US. Patent 0004121 A1, 2009.

(21) Hao J., Chen B., Yao Y. Hossain M. Nagatomo T., Yao H., Kong L., Sun H., Bioorg. Med. Chem. Lett., 2012, 22, 3441.

(22) Descours D., Festal D., Leger J.M., Carpy A., Helv. Chim. Acta, 1991, 74, 1757.

(23) Blasioli C., Heymes A., EP. 0069013 A1, 1982.

(24) Ebbers E.J., Ariaans G. J.A., Zwanenburg B. et.al, Tetrahydron: Asymmetry 1999, 10 3701 .

(25) Jacques, J., Collet, A., Wilen, S.H., Enantiomers, Racemates and Resolutions; Krieger, Malabar, Florida, 1994.

(26) Broughton, D. B.; Gerhold, C. G. Continuous sorption process employing fixed bed of sorbent and moving inlets and outlets. US. Patent 2,985,589, 1961.

(27) Ruthven, D. M.; Ching, C. B., Chem. Eng. Sci. 1989, 44, 1011.

(28) Seidel-Morgenstern, A.; Kessler, L. C.; Kaspereit, M., Chem. Eng. Technol., 2008, 31, 826.

(29) Balawejder M., Gałan K., Elsner M. P., Seidel-Morgenstern A., Piątkowski W., Antos D., Chem. Eng. Sci., 2011, 66, 5638-5647.

(30) Balawejder M., Kiwala D., Lorenz H., Seidel-Morgenstern A., Piątkowski W., Antos D., Cryst. Growth Des., 2012, 12, 2557-2667. 
(31) Olbrycht M., Balawejder M., Matuła K., Piątkowski W., Antos D., Ind. Eng. Chem. Res., 2014, 53, 15990-15999.

(32) G. Guiochon, A. Felinger, D.G. Shirazi, A.M. Katti, Fundamentals of Preparative and Nonlinear Chromatography, Elsevier, New York, 2006.

(33) G. Ziomek, D. Antos, L. Tobiska, A. Seidel-Morgenstern, J. Chromatogr. A, 2006, 1116, $179-188$. 


\section{APPENDIX}

To calculate the mass and the composition of the crystalline phase and mother liquor in each stage $k$-th the following mass balance equations have to be solved: ${ }^{29-31}$

$$
\begin{aligned}
& S_{k-1}+S I_{k}=M_{k} \\
& S_{k}+L_{k}=M_{k} \\
& S_{k-1} x_{i, k-1}^{S}=M_{k} x_{i, k}^{M} \\
& S_{k} x_{i, k}^{S}+L_{k} x_{i, k}^{L}=M_{k} x_{i, k}^{M} \\
& \sum_{i=1}^{2} x_{i, k}^{S}=1
\end{aligned}
$$

The SLE relationship is described by functional dependencies in the following form:

$$
x_{i, k}^{L}=f\left(x_{2,4, k}^{S}\right) \quad i=\left(2 R, 2^{\prime} R\right) ;\left(2 S, 2^{\prime} S\right) \text { or }\left(2 R, 2^{\prime} S\right) ;\left(2 S, 2^{\prime} R\right)
$$

where $S_{k}, L_{k}, S_{k}$ - mass of crystalline phase, mother liquor, and solvent added in $k$-th stage ( $k$ $=0$ denotes feed, $F$ ), respectively; $M_{k}$ - mass at the mixing point (the solution obtained after mixing the phases); $x_{i, k}^{L}$ denote mass fraction of the pair $\mathbf{1 , 3}$ or $\mathbf{2 , 4}$ in the mother liquor in the equilibrium with the mass fraction, $x_{i, k}^{S}$ in the crystalline phase.

Moreover, to facilitate recycling the mother liquors $d_{2,4}$ of $L_{k}$ withdrawn form for $k>1$ was set to be equal to that delivered to the stage $S_{k-2}:{ }^{29-31}$

$$
\bar{X}_{2,4, k}^{L}=X_{2,4, k-1}^{S}
$$

where: $\bar{x}_{2,4}^{L}=x_{2,4}^{L} /\left(x_{2,4}^{L}+x_{1,3}^{L}\right)$

$$
x_{1,3}^{L}=-0.0892\left(x_{2,4}^{S}\right)^{3}+0.2402\left(x_{2,4}^{S}\right)^{2}-0.2182 x_{2,4}^{S}+0.0727
$$

The set of equations (A1-A7) has only one free parameter, i.e., one value for all the masses and the compositions can be set. If, for instance, the product purity is set, the set of equations can be solved providing only one solution. A detailed description can be found in the previous studies. ${ }^{29-31}$ 


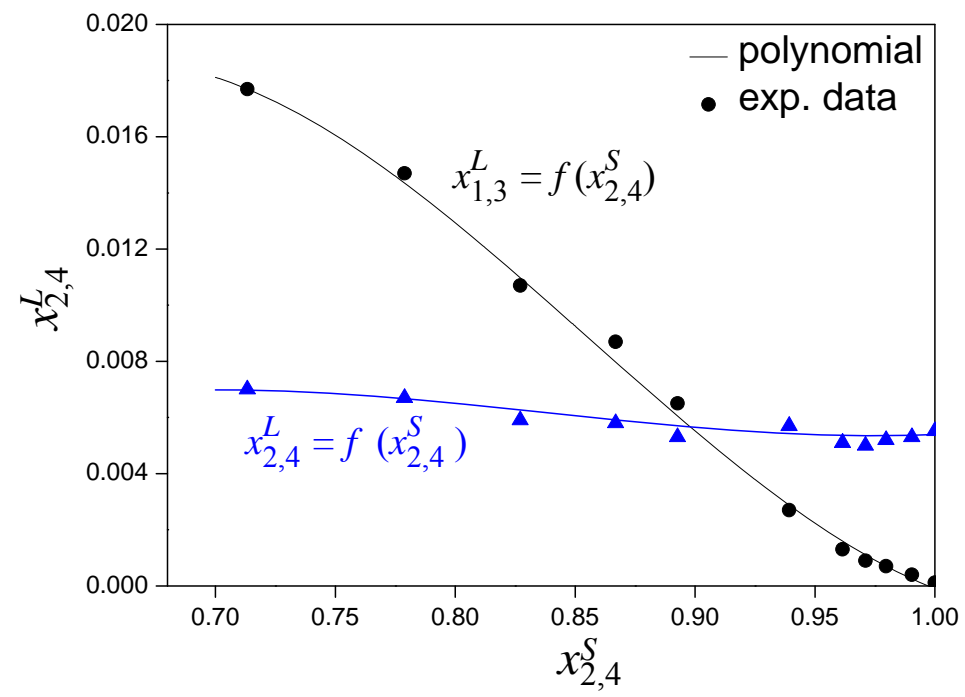

Figure A1. Illustration of the SLE relationship for nafronyl oxalate salt at $20^{\circ} \mathrm{C}$ 